\title{
MEMRISTORS
}

\section{A-maze-ing arrays}

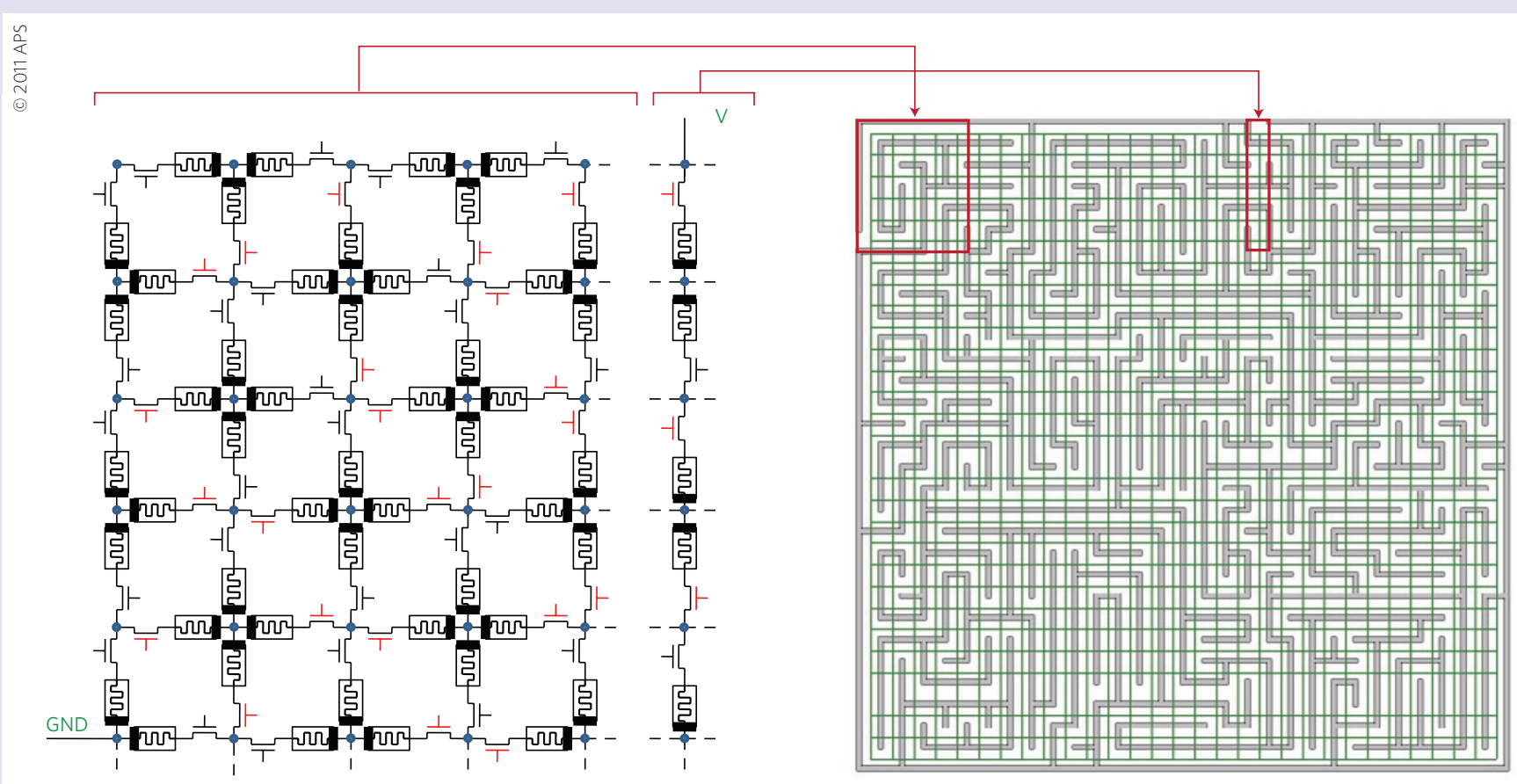

Calculating the shortest path between the beginning and end points of a maze is a classic problem in mathematics. Many algorithms exist for solving this problem, but they become inefficient and slow as the size and complexity of a maze increases. Yuriy Pershin and Massimiliano Di Ventra show that with an array of memristors this and similar calculations that are computationally intractable to conventional computers could become trivial (Phys. Rev. E 84, 046703; 2011).

A memristor is an electronic circuit element that behaves as a resistor with memory. The concept was first proposed 40 years ago by Leon Chua (IEEE Trans. Circuit Theory 18, 507-519; 1971). Chua posited that given that there were fundamental circuit elements to control changes in voltage with current (resistors), charge with voltage (capacitors), and magnetic flux with current (inductors), there should be a fourth element that controls flux with charge. The upshot of this is that the resistance of such an element depends on the history of the current that flowed through it. That is, it has a memory of its previous state - a state that can be switched between high resistance, 'off', and low-resistance, 'on', states - hence the name.

But although all real components exhibit some 'memristance' - just as all components exhibit some degree of resistance, capacitance and inductance - only very recently have device elements with non-negligible memristive responses been realized (Nature 453, 80-83; 2008). These devices consist of a thin titanium oxide film sandwiched between two metal electrodes, with a high concentration of charged, mobile oxygen vacancies distributed near one of the electrodes. Passing a current through the device drives these vacancies further into or away from the centre of the oxide, depending on the direction of the current, decreasing or increasing its resistance, respectively. And when the current is switched off, the movement of vacancies ceases, allowing the device to remember its resistance state.

Unlike more conventional circuit elements, the characteristics of memristors improve as their size is reduced. This fact combined with their simple structure and operation makes them a promising potential basis for non-volatile digital memories. A recent announcement by Stan Williams, Senior Fellow at Hewlett-Packard, suggested that commercial memristor memories could be on the market within two to four years.

Hot on the heels of this, Pershin and Di Ventra show that memristors could also lead to a powerful new approach to massively parallel computing. They simulated a large memristor array, and by switching individual elements of this array on or off were able to mimic the structure of a large complex maze (pictured). By simply applying a voltage between the entry and the exit points of this maze, simple circuit theory predicts that the flow of current will naturally trace the shortest path - a single computational step, independent of the size of the maze. In contrast, the time taken for conventional computer algorithms to find this path increases as the square of the size of the maze. The authors suggest that a similar approach could be applied to even more challenging tasks, such as the travelling salesman problem and other computationally hard problems.

ED GERSTNER 